\title{
An Automated Visual Recognition System to Counteract Illegal Dumping in Smart Cities
}

\author{
Mauro Coccoli \\ DIBRIS \\ University of Genoa, \\ Genoa, Italy
}

\author{
Vincenzo De Francesco \\ DIETI \\ Federico II Naples University, \\ Naples, Italy
}

\author{
Antonio Fusco \\ Asia Napoli S.p.A. \\ Naples, Italy
}

\author{
Paolo Maresca \\ DIETI \\ Federico II Naples University, \\ Naples, Italy
}

\begin{abstract}
In this paper we will describe the prototype form of an automated visual recognition system designed to mitigate illegal dumping, as the outcome of an experiential learning activity. The presented solution relies on the sensor networks of a smart city where the waste management system is supposed to be integrated with other municipality services for environment control and management. In particular, we want to take advantage of the pictures, frames and videos continuously recorded by cameras installed in the cities for traffic monitoring, for surveillance or any other reason. Such data are processed by means of cognitive computing techniques and a specific algorithm of image analysis has been trained to identify trash, especially bulky waste, where it should not be, and trigger an alarm to the municipality. Besides, an organization plan is also proposed for intelligent waste collection as well as some organizational ideas for scalability. The learning activity has been conducted within the program "Party Cloud Challenge per Genova" promoted by IBM in collaboration with the city municipality of Genoa, Italy.
\end{abstract}

Keywords-smart city; cognitive computing; image recognition; waste management; environment

\section{INTRODUCTION}

Commonly, the idea of smart city is tied to using novel ICT solutions to develop mobile intelligent applications to provide effective services seamlessly integrated with the main city infrastructures and sensor networks for environment control and management. However, we must remember that this general view should include technological aspects as well as people and institutions. In fact, the adoption of sophisticated systems and advanced solutions are just enablers for the institutions to set active policies driven to enhance citizens' quality of life. Moving along these three main dimensions, the objectives of a smart city can be summarized as the following: (i) integration of infrastructures and technology-mediated services, (ii) social learning for strengthening human infrastructure, and (iii) governance for institutional improvement and citizen engagement [1]. In this respect, most projects for making a city smart, result in the definition of new models to mitigate current urban problems, to the aim of improving services to the citizens and, thus, making cities attractive places to live in. From a practical point of view, most smart cities implement a common reference model that addresses global sustainability challenges at a local level [2]. Regardless what are the implementation details, the available sensors, the installed instrumentations, the adopted interconnection techniques, the integration methodologies, the ways for exchanging data, possibly in open data format, and the number of possible applications, the main topics addressed in designing smart cities services are edemocracy, e-health, sustainable urban mobility, water, energy, environment, pollution. In this flavor, triggered by the initiative "IBM Party Cloud Challenge per Genova" -Italian for "for Genoa'- this paper focuses on the use of cognitive computing techniques to develop an automated alerting system based on visual recognition, to support the municipality of the city of Genoa, Italy, in counteracting illegal dumping of bulky waste in the streets. The developed system was used as a demonstrator in a Bachelor's Degree thesis in computer engineering, centered on the use of cognitive computing technologies and design methodologies, to show how they can accelerate and improve the learning processes in universities [3, 4].

Applying the smart city paradigm to the field of waste management is an important topic for the environment control and management in urban settings, and poses challenging issues. To find effective solutions, we explore the possibility of using novel cognitive computing technologies. Generally speaking, waste management refers to finding solutions and advanced techniques to manage the garbage lifecycle and disposal processes such as, e.g., using sensorized bins in an Internet of Things (IoT) framework [5] to optimize pickup operations and transports to decentralized dumps. Nevertheless, a smart waste management should embrace an integrated planning strategy tailored for resource recovery and efficiency within a circular economy framework [6]. The easiest and more economical solution is calling people to the action of reducing the volume of the rubbish they produce, also encouraging a careful separate collection of waste. Anyway, due to the possible presence of non-polite citizens, we also have to consider policies aimed to the reduction of illegal dumping of bulky waste. An alternative and sophisticated way to achieve this objective is using the large amount of data already available within a smart city environment owing to the installed sensor networks. Hence, we regard the smart city as a generator of images, coming from different sources, such as, e.g., the videos collected around by many cameras installed by the municipality and security agencies as well, for purposes other than dumping surveillance. Recalling the three-dimensional model cite above, our system implements

DOI reference number: 10.18293/DMSVIVA20-006 
the following: (i) video surveillance system are integrated within the whole smart city framework and data circulate between applications; (ii) given the presence of an automated alerting system, citizens are encouraged to not-dumping illegally; (iii) the city government can take specific actions with dedicated prevention policies. Specifically, we developed an algorithm to generate alerts when a bulky waste is abandoned outside of authorized disposal points. In the following, we present the project submitted to the Challenge per Genova, which was awarded for the first prize (see requirements and evaluation criteria in Section 2).

The remainder of the paper is structured as follows: Section 2 describes the general framework of the Challenge per Genova initiative, while in Section 3 the proposed smart alerting system is presented. Following the implementation details, Section 4 discusses possible impact of the proposed solution in a smartcity framework. Finally, Conclusions and a glance on future works are reported in Section 5.

\section{ThE “ChALLENGE PER GENOVA” INITIATIVE}

As anticipated, this paper reports the experience carried on during the so called "IBM Party Cloud Challenge per Genova". More precisely, the "IBM Party Cloud" is an online hackathon promoted by IBM to accelerate the technological innovation made in Italy at the service of sustainability. The "Challenge per Genova" track was launched on September 20, 2018 in Milan, lasting November 11, 2018, and it was open to developers, data scientists and IT people in general. This specific initiative was supported by IBM Italia, Dock Joined in Tech, Lifegate and Codemotion, under the patronage of the Genoa Municipality and its local inhouse garbage company. In fact, the objective of the hackathon was that of finding novel solutions to counteract illegal dumping more effectively, especially with reference to the disposal of bulky waste in unauthorized places in the city streets and in urban areas in general. Specifically, the statement of the problem posed by the challenge is that, presently, most critical situations are spontaneously reported by citizens, even if more than 300 surveillance cameras are installed all over the town. The video surveillance system requires a number of operators to watch videos to find possible abuses and this is not an efficient way of using human resources. Hence, the objective is developing a smart alerting solution able to find in real-time abuses and promptly trigger alarms to the operation center, which can manage timely counteractions to solve issues arisen. In more detail, from the technical point of view, the participants to the contest are called to develop an application prototype using the IBM Cloud platform. In such an integrated environment, designers and developers are provided with advanced image recognition tools and analytics services, which can be used to identify the abuses described above and, consequently, trigger alarms to signal them via SMS and/or other means. Moreover, any alert is required to include the following: (i) picture of the evidence, (ii) geo-localization coordinates, (iii) type of bulky waste and its spatial features to evaluate its possible dangerousness, (iv) possible information on "who" made the illegal dumping, e.g., a person as well as a vehicle and its plate number, $(v)$ abnormalities, if any.

After the deadline for submitting the projects, the developed applications were evaluated based on the following criteria: (i) usefulness and value, (ii) bearing on the challenge issue, (iii) technical soundness and accuracy, including architectural blueprints, (iv) interface design and user experience, (v) creativity and novelty.

\section{The Proposed SMART AleRting Sysytem}

Given the strict system requirements specified within the framework of the challenge, the structure of the automated alerting system can be described with two functional blocks: (a) identify and classify a possible item of interest in a specific area under video surveillance, and (b) managing the alerting system. To achieve these results, we were provided with a selection of videos recorded by the local surveillance system of the Genoa Municipality, showing non-fictional scenes of illegal dumping actions of some bulky waste. By using consolidated techniques for comparing frames, it is easy to find suspect changes in the scene (see, e.g., [7] and references therein), i.e., the stable presence of a new object in the landscape. Such new and unknown object could be a car in a long-lasting stop as well as an object candidate to be considered a bulky waste, possibly abandoned by the suspect vehicle. When such conditions occur, an operator is called to perform a visual analysis of the video, which may lead to the identification of a possible abuse. We want to make this process unattended, implementing an automated visual recognition system able to identify the presence of bulky waste in a given scene and, consequently, trigger alerts.

Owing to the advanced cognitive computing capabilities offered by IBM Watson within the IBM Cloud platform, all the necessary tools for the development of a software prototype were available. Among these, we used: data analysis and visualization, data preparation and modeling, machine learning capabilities and visual recognition. The result is a system able to identify and classify in real time the bulky waste in unauthorized places and to manage alerts triggering. Specifically, for the development of the application prototype, we adopted Watson studio, which provides the environment and tools to accelerate infusion of artificial intelligence (AI) in applications. In more detail, we used the IBM Watson Visual Recognition service, which has suited algorithms to analyze images and to build a personalized model so to extract meaning from visual content. Figure 1 depicts the relevant reference architecture.

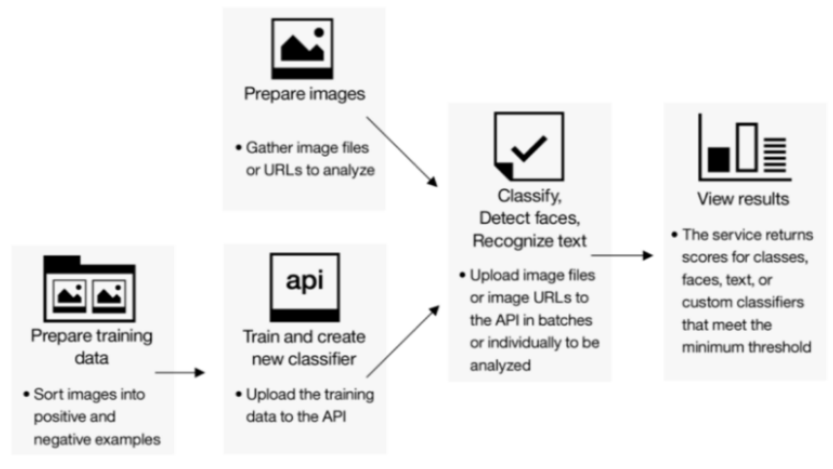

Figure 1. The architecture of Visual Recognition services within the IBM Watson Studio platform. 


\section{A. Visual Recognition and Classification}

As a starting point, we designed our solution based on sampling videos and extracting frames, to the aim of creating a customized visual recognition model, able to identify and classify bulky waste in a given area. According to this model (Fig. 1), the supervised learning process for the recognition model is crucial and, depending on its accuracy, the application may fail or succeed. Hence, for the custom model to work effectively, an accurate training phase is needed for optimization and to guarantee good performances for the classifier, in terms of precision. Such a process is performed as described in Figure 2 , showing the steps needed for creating and training a specialized visual recognition classifier. More precisely, the following Steps 1-3 are repeated recursively:

Step 1 - prepare training images. Gather files to use as positive and negative data example;

Step 2 - create and train the classifier. Specify the location of the training images and call the Visual Recognition API to create the custom classifier;

Step 3 - test the custom classifier. Classify images with the new custom classifier and measure the classifier performance.

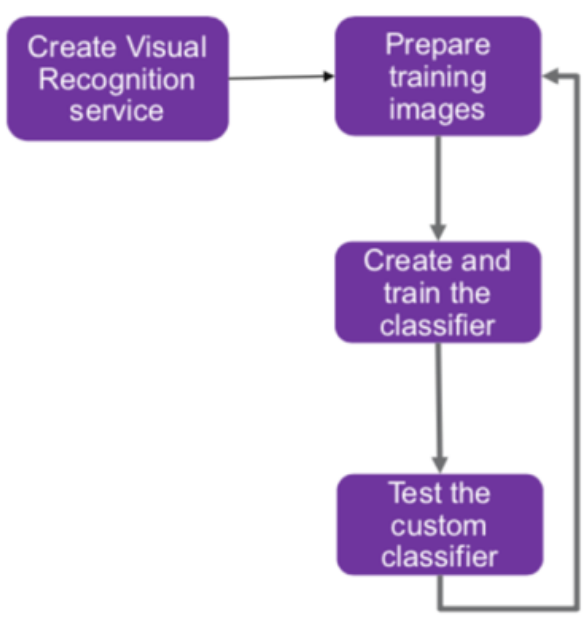

Figure 2. The recursive steps for training the visual recognition classifier.

To effectively perform this task, we have identified the types of waste that are dumped illegally more often, also matching all the ones included in the sample videos. This resulted in the definition of thirteen classes, i.e.: Refrigerator, Washing machine, Couch, Furniture, Freezer, Bicycle, Armchair, Mattress, Chair, Printer, Television, Bed base, and Heap. Then another class has to be added, that is the Negative one, for images that do not depict the visual subject of any of the positive classes, thus containing every non-classifiable item. For each of the above classes, many similar but heterogeneous pictures have been used to train the system, chosen e.g., with different values of brightness and contrast, taken from different angles and in varied light conditions.

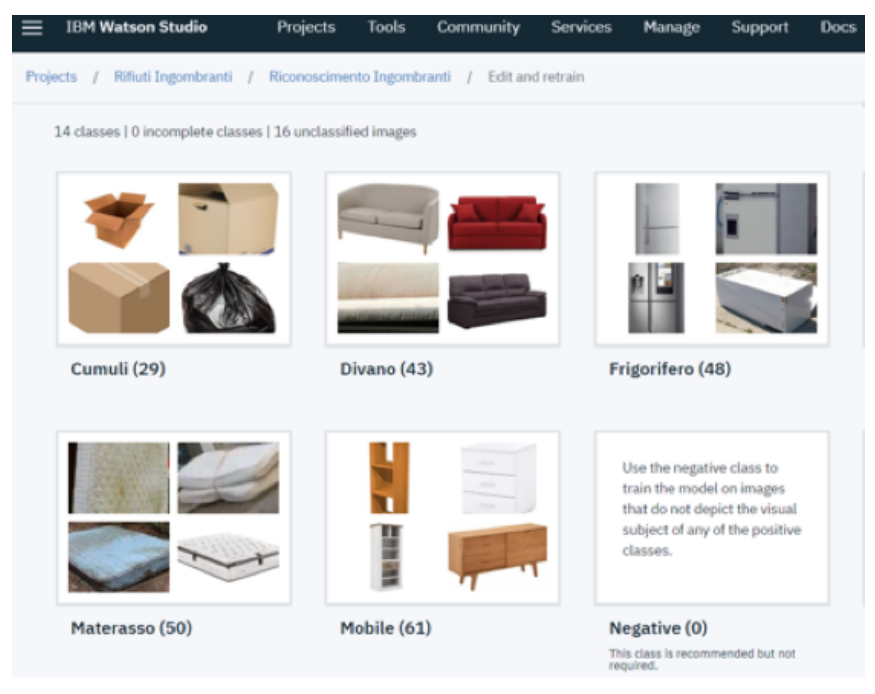

Figure 3. Results of the tests performed to evaluate the visual recognition system after its training phase.

Figure 3 shows a screenshot taken from the Watson Studio Visual Recognition working environment, presenting the results achieved for some of the considered classes, after a preliminary training phase. Please note that the system was programmed in Italian so, for a better understanding, consider the following Italian-English legenda when reading the picture: Cumuli Heap; Divano - Couch, Frigorifero - Refrigerator, Materasso Mattress, Mobile - Furniture. The numbers in parentheses specify the number of sample pictures associated to each class. Then, the real effectiveness of the training phase was tested against both the frames extracted from the original sample videos and generic pictures retrieved through common image search engines available over the Web such as, e.g., Google Images, always using the abandoned prefix in the search keys. For each of these pictures, the classifier assigned a matching score to the classes. Please note that such score is a number between 0 and 1 , which estimates the model's confidence in the classification, based on the used training set, not an absolute percentage of accuracy.

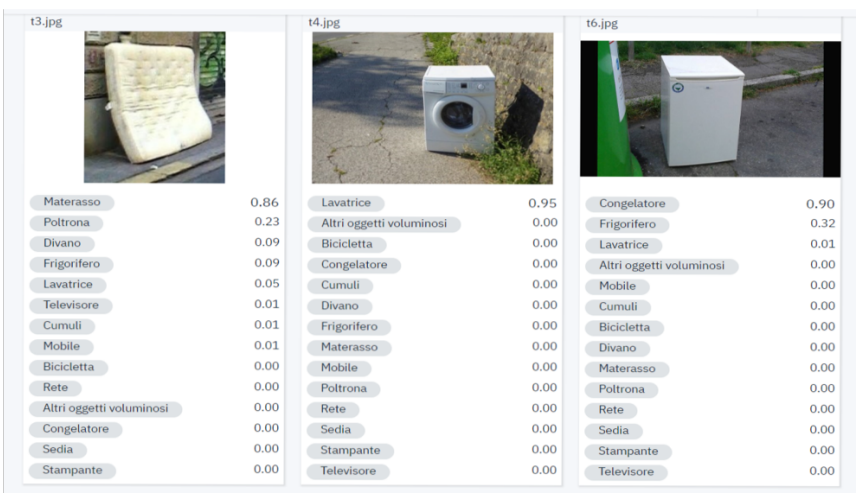

Figure 4. Results of the test on the trained system. 
According to the results shown in Figure 4, we conclude that the custom classifier should be considered reliable. For a better understanding, again, an Italian - English legenda is required, that is the following: Materasso - Mattress, Lavatrice Washing machine, Congelatore - Freezer.

\section{B. The Alerting System}

When in working conditions, the visual recognition system described in the previous sub-section will be fed with frames extracted at a given sampling time from video surveillance cameras. When a suspect item is identified in at least two consecutive frames of the same scene, an alert is triggered, reporting the class for the suspect item and the relevant confidence score to evaluate the accuracy of the alert. Then, the alert message is completed with metadata attached to the video source, such as a unique video identifier, the timestamp through date and time, the geographical localization through coordinates and street address.

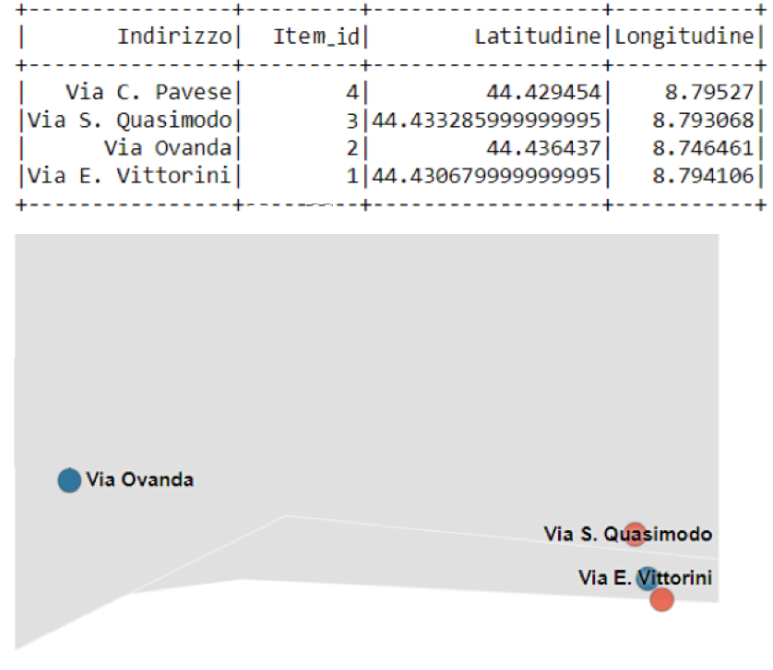

Figure 5. Graphical representation of alerts on a map of the city of Genoa.

More precisely, the alerts are stored in a database table whose fields are the following (names are reported in English and in the corresponding Italian, when used):

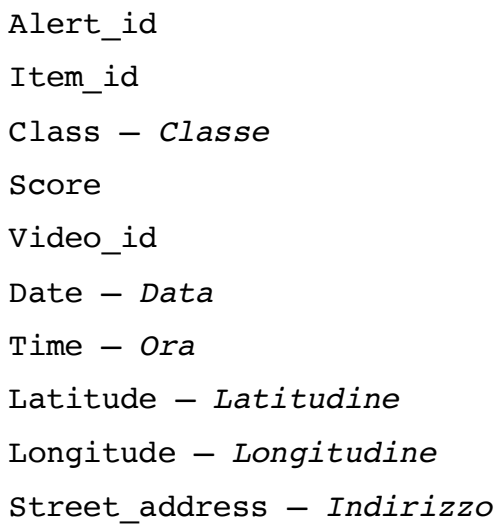

When an alert is received the municipality can launch different procedures in reaction, such as, e.g., reporting to another competent authority (e.g., when a license plate is identified in the images and a vehicle has to be investigated) or collecting the waste.

In addition, the data collected within the analysis of the video frames also allow analyzing different aspects. For example, from an operational point of view, we can identify the position and dangerousness of the abandoned object. Other applications are possible, e.g., the analysis of social impact, the subsequent implementation of precise investigation criteria and, finally, in terms of implementing interventions addressed to prevent citizens to adopt such an undesired behavior. To provide an example, the data are processed with a Jupyter Notebook, one of the data science tools included in the Watson Studio, enabling interactive data analysis and visualization. A specific Python Notebook has been programmed to represent the distribution of alerts on a simplified map of Genoa, as shown in Figure 5.

\section{POSSIBLE IMPACT}

According to the three dimensional smart-city model already mentioned in the introductory section [1], in the following we report some important considerations relevant to:
(a) prospective technology enhancements,
(b) social aspects,
(c) governance.

\section{A. Prospective Technology Enhancements}

What happens after the system triggers alerts with the relevant information is out of the scope of the developed prototype, which should be duly integrated at the business intelligence level of the existing legacy system through suited interfaces and specific middleware. Moreover, another block in cascade should be able to exploit profitably the information transferred by the classifier, possibly with semantic capabilities. For example, in the case the item identified in a bulky waste dumping event is a TV, it should be associated to a specific hazard class, i.e., the RAEE in Italy, equivalent to Electric and Electronic Equipment Waste, which is not considered in our model, due to limitations imposed by the strict timing and the original requirements of the challenge. This should classify the item on the basis of the subsequent pickup procedure and recovery. Furthermore, other data can drive the decisions. For example, the alert date and its persistency allow going back to the number of days of distress to the community due to the presence of the abandoned object. In this case, the collection system should keep into account a priority order in the organization of the service and logistics by choosing, e.g., the suited vehicle for both the characteristics of the item to be uploaded and the width of the street, that, in Genoa and elsewhere in Italy, can be very narrow. Furthermore, using the geographical coordinates can help calculating optimized routes of the collection paths on the road graph, to plan more efficiently the collection sequence and the overall execution times of the extraordinary withdrawal operations. Future developments should also consider security and privacy issues. 


\section{B. Social Aspects}

From the point of view of the social impact, the spatial distribution and the identified commodity types allow to construct a dynamic map of the distribution and incidence of the abandonment phenomena, with special attention, e.g., to suburbs. Assuming a principle of proximity at the base of the phenomenon, timely communication and awareness campaigns for users can be started in order to discourage the recurrence of such phenomena. Furthermore, it is possible to or predict periods of the year in which such illegal dumping phenomena occur more often. This allows planning more frequent controls or other specific actions e.g., with more frequent collection services or making available new cumbersome stuff transfer facilities under a controlled regime, which may be both fixed or temporary, i.e., itinerant vehicles.

\section{Governance}

Both the above-described aspects are of paramount importance to reduce the impact and the incidence of the phenomena of bulky waste illegal dumping in cities. In fact, the economic and social advantages that may derive from the resolution of this phenomenon are far superior to the costs of activation of specific prevention programs and of a reorganization of existing services. The prototype presented in this paper, contributes first of all to enable a wider understanding of the phenomenon and its causes, on an analytical basis, which may be due to the lack of collaboration of the citizenry and/or the inadequacy of the relevant policies and planned services. In both cases, the availability of a database of reports on a geographical basis, with a precise classification of the abandoned waste, makes it possible to design and test targeted interventions to optimize the resources devoted to solve the problem, very felt by the community in terms of overall perception of the quality of the service provided by the civic operators.

\section{CONClusions AND FutURE WORK}

Following the Challenge per Genova initiative, we faced a specific issue of the waste management problem, which requires more complex solutions and the integration of different interoperable systems. Specifically, we focused our effort on developing and testing of an automated visual recognition system based on cognitive computing, and on collecting data and metadata to transmit with alerts, according to the given requirements. As we try expanding the boundaries of the problem analysis, several possibilities arise to extend the basic functionalities of the developed prototype. Depending on the observed filed of application, improvements can be done in different directions, to achieve different objectives. In the following we report three examples of possible enhancements:

(i) expanding the set of data collected, managed and processed, through a suited optimization of the presented visual recognition system. In fact, fully exploiting the cognitive computing capabilities available, we can estimate the overall geometrical characteristics of the detected items both in terms of dimensions (linear and volumetric) and in shape. Moreover, the simultaneous recognition of multiple objects in a single frame or at least the identification of the prevailing commodity fraction would be a desirable feature. With such information available, alerts would include the GPS coordinates of the interested place as well as the expected number of objects to be collected there, including their type, size, and possible dangerousness;

(ii) optimizing the collection path design, based on the information provided by the alerts for any point an abandoned item is detected in. A possible solution is performing a classification for the collection points, according to the gravity of the situation and the numerosity of waste and their dimensions and expected weight; as well as for the time spent from the alert. This can result in a priority-based list of sites to be visited and give hints on the best suited vehicle for safe collection and transport operations;

(iii) creating an interactive reporting service so that users, i.e., citizens, can signal abuses, taking pictures and uploading them with data such as, e.g., a description of the object, precise position with address, other useful information, including personal ones. The same interface can also be exploited to prevent illegal dumping by implementing a pick-up service on-demand, in the case a cumbersome object has to be trashed by any citizen.

As a final remark, we observe that this work is also relevant from the educational point of view, for it copes with emerging needs of both the modern society and the labor market, driving the evolution of the current higher education model. In fact, this activity can be regarded as an example of experiential learning, where knowledge is created through the transformation of experience [8]. Such a learning methodology requires students to actively acquire knowledge by doing and applying their own problem-solving abilities [9]. As students are actively involved in the learning process, their learning satisfaction increases as well as their understanding and retention of course material, and they voluntarily become continuous learners, also improving interpersonal communication skills, as well as developing analytical thinking, and critical thinking abilities [10]. In particular, for the specific case of software engineering classes, examples of laboratory experiences involving third parties can be found, e.g., in [11] and references therein.

\section{ACKNOWLEDGMENT}

The research has been conducted in the metropolitan area of the city of Genoa, Italy, within the program "Party Cloud Challenge per Genova" promoted by IBM Italia, Dock Joined in Tech, Lifegate and Codemotion, under the patronage of the Genoa Municipality and AMIU, the local inhouse garbage company.

\section{REFERENCES}

[1] T. Nam and T.A. Pardo, "Conceptualizing smart city with dimensions of technology, people, and institutions," in Proceedings of the $12^{\text {th }}$ Annual International Conference on Digital Government Research: Digital Government Innovation in Challenging Times, College Park, Maryland, USA, June 12-15, 2011, pp. 282-291.

[2] S. Zygiaris, "Smart city reference model: assisting planners to conceptualize the building of smart city innovation ecosystems," in Journal of the Knowledge Economy, no. 4, 2013, pp. 217-231. 
[3] M. Coccoli, P. Maresca, and L.Stanganelli, "Cognitive computing in education," Journal of e-Learning and Knowledge Society, vol. 12, no. 2, 2016, pp. 55-69.

[4] M. Coccoli, P. Maresca, and L.Stanganelli, "The role of big data and cognitive computing in the learning process," Journal of Visual Languages and Computing, vol. 38, 2017, pp. 97-103.

[5] T. Anagnostopoulos, A. Zaslavsky, K. Kolomvatsos, A. Medvedev, P. Amirian, J. Morley and S. Hadjieftymiades, "Challenges and opportunities of waste management in IoT-enabled smart cities: A survey," IEEE Transactions on Sustainable Computing, vol. 2, no. 3, 2017, pp. 275-289.

[6] A. Del Borghi, M. Gallo, C. Strazza, F. Magrassi and M. Castagna, "Waste management in smart cities: The application of circular economy in Genoa (Italy)," Impresa Progetto Electronic Journal of Management, vol. 4, 2014, pp. 1-13.
[7] H. F. Ng and C. Y. Chin, "Effective scene change detection in complex environments," International Journal of Computational Vision and Robotics, vol.9, no. 3, 2019, pp. 310-328.

[8] D.A. Kolb, Experiential learning: Experience as the source of learning and development. Prentice-Hall, Inc. Englewood Cliffs, NJ, 1984.

[9] M. McCarthy, "Experiential learning theory: From theory to practice," Journal of Business \& Economics Research (JBER), vol. 14, no. 3, 2016, pp. 91-100.

[10] D. R. Brickner and E. R. Etter, "Strategies for promoting active learning in a principles of accounting course," Academy of Education Leadership Journal, vol. 12, no. 2, 2008, pp. 87-93.

[11] M. Coccoli, P. Maresca, L. Stanganelli and A. Guercio, "An experience of collaboration using a PaaS for the smarter university model," Journal of Visual Languages and Computing, vol. 31, 2015, pp. 275-282. 